\title{
Occipital Dermoid Cyst Associated with Dermal Sinus and Cerebellar Abscesses
}

\author{
Gurdeep S. Mann, Ashok Gupta, D. Douglas Cochrane, Manraj K.S. Heran
}

Can. J. Neurol. Sci. 2009; 36: 487-490

Extradural dermoid cyst with an intact occipital dermoid sinus is a rare entity, with fewer than 20 cases reported in the literature $^{1}$. We describe the unique computed tomogram (CT) and magnetic resonance (MR) imaging findings of an infant presenting with cerebellar abscesses and secondary obstructive hydrocephalus resulting from an occipital dermoid cyst with a complete dermal sinus, confirmed at operation. Early neurosurgical intervention is advocated to prevent the ongoing risk of significant complications, including cerebellar abscess formation, hydrocephalus and bacterial meningitis. The importance of cross sectional imaging in the assessment of dural venous sinus anatomy in relation to the pre-operative planning of posterior fossa dermoid cysts is discussed.

\section{Case History}

A previously healthy 20 -month-old female initially presented with two short and self-limited episodes of low grade fever and vomiting associated with non-specific pain and irritability.

She presented two weeks later to an outside institution with lethargy and low-grade fever, for which oral amoxicillin was prescribed. Four days later, she became more lethargic, refusing to walk or feed, and began to drool, with increasing irritability and intermittent non-bilious vomiting. She was re-admitted and noted to have a white blood count of 12.5 and elevated Erythrocyte Sedimentation Rate of 85 with no obvious cause identified. She initially improved but rapidly deteriorated, with obvious head tilt, lethargy, vomiting and ataxia. No focal neurological deficit was elicited.

Cranial CT examination was performed (Figure 1) showing a well defined, midline occipital serpentine bony tract directly communicating with a right cerebellar hemispheric mass lesion. Following intravenous contrast, the mass lesion showed several thick walled ring enhancing cysts and effacement of the fourth ventricle with accompanying non-communicating hydrocephalus, trans-ependymal flow and transtentorial herniation. Cranial CT venography confirmed the bony defect coursed directly underneath and through the inferior margin of the torcular Herophili and could not be clearly separated from the adjacent dural venous sinuses.

A cranial MRI examination was performed under sedation to better delineate the lesion and the surrounding venous anatomy (Figure 2). Unfortunately the patient's clinical condition rapidly deteriorated during scanning, necessitating immediate neurosurgical intervention prior to performing dedicated MR venography.

Cranial MRI confirmed the CT findings. Several ring enhancing cysts were identified in the posterior fossa showing mass effect and containing fluid-fluid levels, with intermediate signal contents on $\mathrm{T} 1 \& \mathrm{~T} 2$ weighted imaging, and restricted diffusion, in keeping with pus. Restricted diffusion was evident in the dermal sinus tract, in keeping with pus. Pre-operative examination with the head shaved revealed a midline occipital dermal sinus, corresponding to the CT findings, with a tuft of hair arising from within it. (Figure 3a \& 3b)

Our patient underwent a sub-occipital craniotomy (Figure 3c) and radical surgical excision of the occipital dermal cyst with removal of hair, fluid contents and dermal sinus tract. Passage of the dermoid cyst through the inferior leaf of the torcular Herophili necessitated careful marsupialization. The adjacent dura was markedly deformed as it encapsulated the dermoid cyst, manifesting as a bulging soft tissue horn at its apex just below the torcular.

Aspiration and gross capsular excision of the multiple cerebellar abscesses was performed. The piece of bone around the dermal sinus tract was abnormally thickened, necessitating separate removal.

Histopathological examination confirmed a dermoid cyst arising from the fourth ventricle surrounded by mature central nervous tissue. Hair follicles arising within the cyst had incited a foreign body giant cell response with numerous sebaceous cysts containing cholesterol clefts seen in the periphery. Adjacent to this, a partially keratinized squamous epithelium-lined tract in keeping with a ruptured dermal sinus tract was noted within the dermis extending into the subcutaneous soft tissues.

Organisms grown from the abscess included Propionibacterium avidum, peptrostreptococcus, and enterococcus faecalis. Antibiotic treatment, based on organism sensitivity, entailed four weeks of intravenous penicillin.

The patient's post-operative course and subsequent clinical follow-up to-date have been uneventful.

\section{Discussion}

An occipital dermal sinus terminating as a posterior fossa dermoid tumour was first described by Ogle in $1865^{2}$.

\footnotetext{
From the Department of Radiology (GSM, MKSH), Department of Neurosurgery (AG, DDC), BC Children's Hospital, Vancouver, BC, Canada.

Received November 17, 2008. Final Revisions Submitted March 19, 2009. Correspondence to: Manraj Kanwal Singh Heran, Address: Department of Radiology, BC Children's Hospital, 4480 Oak Street, Vancouver, British Columbia, V6H 3V4, Canada.
} 


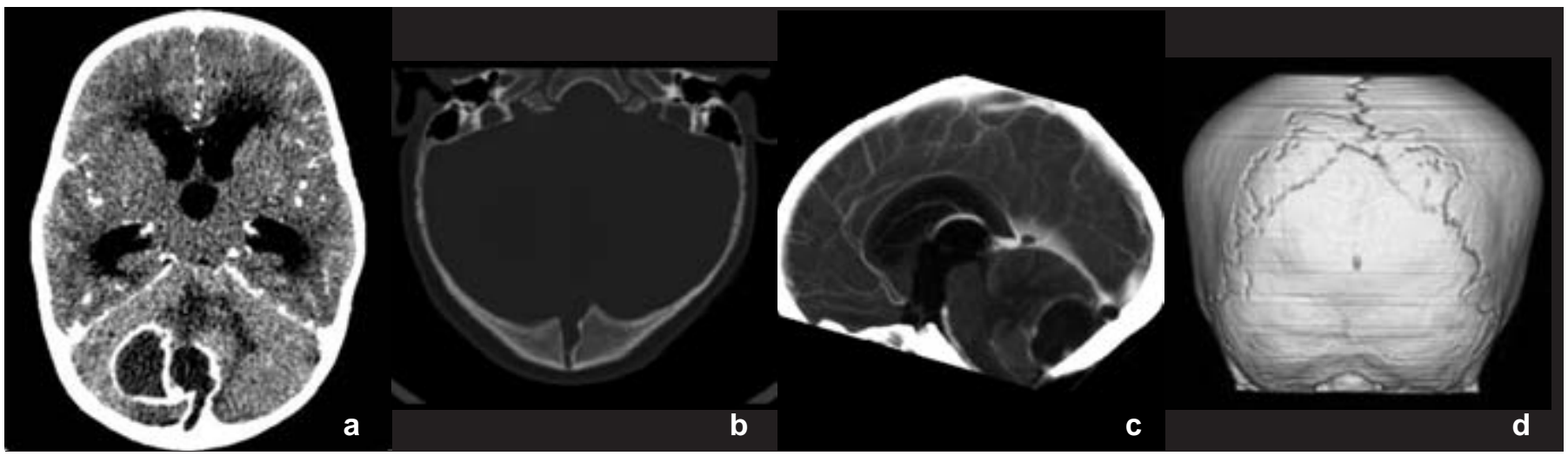

Figure 1: a) Axial contrast enhanced CT showing multiple thick walled cerebellar ring enhancing abscesses and associated compression of the fourth ventricle with accompanying obstructive hydrocephalus and trans-ependymal flow; b) Axial reformatted image windowed to show the irregular midline occipital bony tract; c) Sagittal reformatted image demonstrating the posterior fossa abscesses and resulting infratentorial herniation and close proximity of the dermal sinus tract to the torcular Herophili; d) Surface rendered $3 D$ image showing the occipital bone pit.

Intracranial dermoid sinus cysts are a rare benign tumour and account for approximately $0.1-0.7 \%$ of all intracranial tumours ${ }^{3}$.

Dermal sinuses are often clinically occult. The most common association is the presence of a dermoid or epidermoid cyst, and rarely meningoceles and encephaloceles ${ }^{4}$. In the infant, they may be identified by recognizing the presence of a cutaneous pit or a midline subcutaneous mass. Intracranial dermoid sinuses more commonly present in neonates and infancy due to neuromeningeal infection and abscess formation. Local mass effect resulting in raised intracranial pressure with or without hydrocephalus is seen with increasing tumour size, typically in older patients ${ }^{4}$. Secondary infection, which may be recurrent, occurs in the presence of a diminutive dermal sinus tract ${ }^{5}$.

Occasionally, supratentorial dermoid cysts have directly resulted in neurologic dysfunction. Meningism secondary to aseptic meningitis and convulsions have also been described in intracranial dermoid cyst rupture ${ }^{5,6}$.
Various theories exist regarding the embryology of dermal sinuses $^{7,8}$. A congenital dermal sinus is a form of craniospinal dysraphism, which can affect any level of the developing neural axis from the nasion to the conus, most commonly occurring in the lumbosacral region. The second most common site is the occipital squama. Other intracranial sites include the anterior fontanelle and crista galli ${ }^{4}$. They often occur at or just off midline. Whereas nasal dermoid lesions are typically extradural, those situated dorsally are not uncommonly intradural. Occipital lesions are often directed inferiorly, arising from the roof of the fourth ventricle, cerebellar vermis or subdural space, and penetrating the dura to terminate in the occipital epithelium. Dermal sinuses exhibit a variable degree of intracranial penetration with the vast majority reaching the subarachnoid space, infrequently ending in the scalp or skull and rarely in the epidural space 4 .

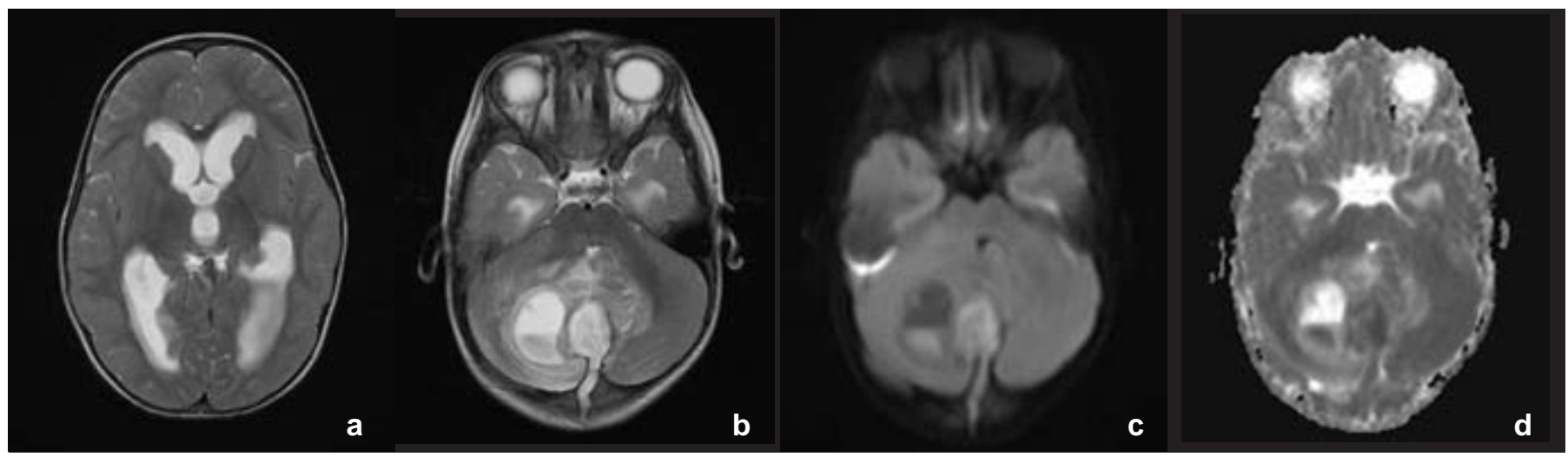

Figure 2: Axial pre-operative MRI images: a) T2 weighted image at the level of the third ventricle demonstrating hydrocephalus and transependymal flow and debris in the left lateral ventricle; b,c,d) T2, DWI (B1500) \& apparent diffusion coefficient images at the level of the fourth ventricle demonstrating restricted diffusion within the occipital dermal sinus tract and within the dermoid cyst and associated abscesses, with a fluid-fluid level within the posterior fossa abscess cavity. 


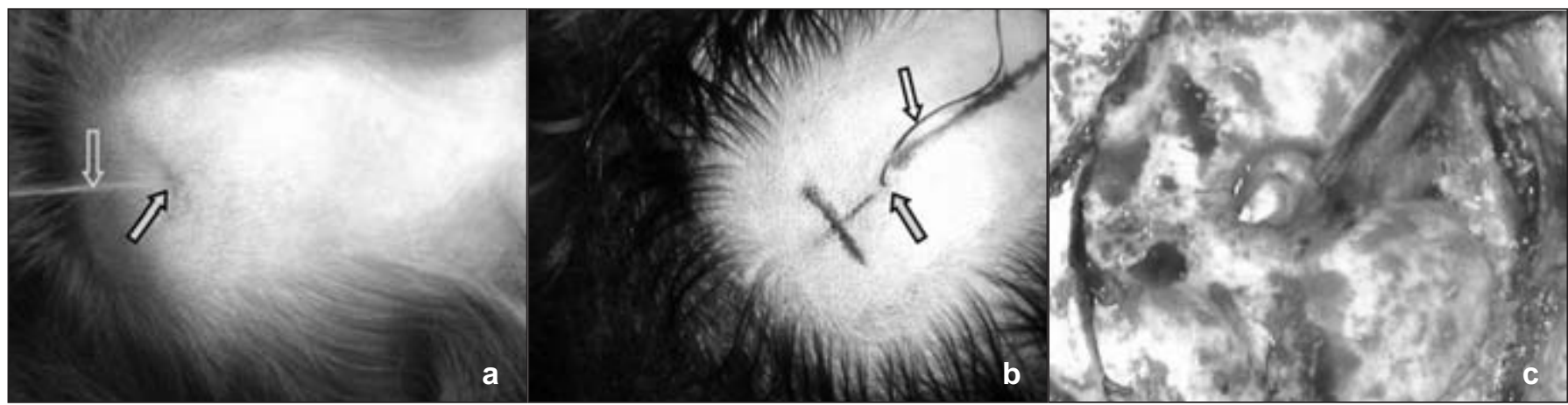

Figure 3: $a, b)$ Pre-operative images of tuft of hair (open arrow) arising from midline dermal sinus tract (closed arrow) ; c) Intra-operative image with a sucker pointing to an extradural tract going through the dura.

Several authors consider a congenital dermal sinus is due to incomplete dysjunction resulting from the failure of fusion of midline elements at three to five weeks of gestational life ${ }^{9,10}$. Dysjunction is the normal separation of the neural tube from the overlying ectoderm. A dermal sinus tract results from abnormal adhesions between the ectoderm destined to form the neural tube from the dermis. Dermal elements either lie adjacent to or are enveloped by the developing neural tube. The dermoid cyst lies at end of the tract. Congenital dermal sinuses arise from primitive ectoderm and contain both epidermal and dermal elements. Epidermal elements include differentiated stratified squamous epithelium. Dermal elements include sebaceous glands, hair follicles, sweat glands and fat. Approximately $60 \%$ end in a dermoid or epidermoid tumour, since ectodermal, or mixed ectodermal and mesodermal elements can be trapped along the tract. Approximately $30 \%$ of such tumours are associated with a sinus tract ${ }^{4}$.

Posterior fossa dermoid tumours have been classified according to location and degree of development of the associated dermal sinus:

(1) extradural dermoid cyst with complete sinus

(2) intradural dermoid cyst without a dermal sinus

(3) intradural dermoid cyst with an incomplete dermal sinus

(4) intradural dermoid cyst with a complete dermal sinus 9

Prompt neurosurgical intervention is often necessary to reverse presenting neurologic deficits, and treat systemic complications from severe intracranial infection secondary to abscess formation or bacterial meningitis. Definitive treatment involves complete removal of the dermal sinus tract and related tumours, and appropriate aspiration, drainage or excision of any accompanying abscesses. No cases of clinical recurrence have been described to date, including cases with incomplete excision $^{9,11}$. There is a single case report of recurrent posterior fossa abscess formation 20 years following surgery for posterior fossa dermoid cyst and bilateral abscesses ${ }^{12}$.

Communicating hydrocephalus from meningitis or noncommunicating hydrocephalus from posterior fossa mass effect have been variously treated with external ventricular drainage, ventriculo-peritoneal shunting, or conservatively, as in our case.
The main risk of surgery is significant bleeding encountered during the dissection of a cyst from the adjacent dural venous sinuses $^{11}$. Fatal exsanguination has rarely been described in the literature ${ }^{13}$. In our case, the dermal sinus tract coursed through the occipital dural venous sinus confluence, necessitating very careful marsupilization.

Various anomalies of the dural venous sinuses have been described in relation to congenital occipital dermal sinus and have included elongation of the vein of Galen, division of the sagittal sinus, elevation of the torcular, elevation of the transverse sinuses, and stenosis of the torcular angle. These findings have also been described in obstructive lesions of the neural tube and in Dandy Walker cyst ${ }^{14}$.

The imaging features of dermoid tumours are variable and largely depend on their fat content. On plain film imaging, a well defined hypodensity may be apparent with occasional calcification enabling differentiation from a lipoma. The associated bony tract may be visible on plain films, but is best delineated by CT. A CT can reveal the exact location of the cyst, its relationship with the adjacent sinus, and any related cranial vault thinning or focal defect. The cyst typically shows homogenous attenuation characteristics with attenuation values ranging between 0 to $150 \mathrm{HU}$, and higher values seen in infected cysts ${ }^{1}$. Unless infected, the cyst does not enhance with contrast. Occasionally, desmoplastic thickening of the adjacent dura shows contrast enhancement ${ }^{1}$. Contrast enhancement of the associated posterior fossa abscesses of an infected dermoid cyst is seen on both CT and MRI.

Dermoids typically show high $\mathrm{T} 1$ and $\mathrm{T} 2$ signal, suppress with short tau inversion recovery (STIR), and, when infected, have restricted diffusion on DWI and ADC imaging. Conventional cerebral angiography typically shows an avascular mass $^{15}$.

Magnetic resonance imaging has been advocated as the imaging modality of choice in assessing dermoid cysts ${ }^{7,11}$. The MR involves no ionizing radiation and has superior soft tissue contrast in comparison to CT. The main disadvantages of MRI are the frequent requirement for sedation or general anaesthesia in the young child and longer scan time.

The role of MRI in evaluating the dural venous sinuses, meninges and cerebellar parenchyma in relation to a dermoid 
cyst has not previously reported ${ }^{12}$. In our case, the exact location of the posterior fossa dermoid cyst could only be determined at the time of operation, in keeping with a previous report ${ }^{12}$.

Previously, MR angiography has been advocated to determine the relation of the confluence of the dural venous sinuses and a midline dermal sinus ${ }^{1,8,12}$. In our case, we were unable to complete the MRA study as intended due to the patient's clinical instability. A good quality venous phase multi-detector CT angiogram was obtained with multiplanar reformatted images, demonstrating its sensitivity in depicting the dermal sinus and proximity of the torcular. Computed tomogram also accurately defined the complications of abscess formation and hydrocephalus.

Posterior fossa dermoid cyst should be considered in any child with an occipital cutaneous lesion.

An intact occipital dermal sinus with an extradural dermoid cyst mandates early neurosurgical intervention to prevent the increasing risk of the sequalae of intracranial infection. Preoperative cross sectional imaging is essential to assess the dermal sinus tract, optimize the surgical approach, and to assess for any related complications. Like others, we continue to advocate the use of MRI/MRA to evaluate the proximity of dural venous sinuses to mitigate the high risk of significant bleeding at operation. In the critically unwell patient requiring urgent intervention, we have found contrast enhanced multi-detector CT, with its inherent multiplanar capabilities, to rapidly provide similar clinical information, making this a useful alternative to MR.

\section{REFERENCES}

1. Akhaddar A, Jiddane M, Chakir N, El Hassani R, Moustarchid B, Bellakhdar F. Cerebellar abscesses secondary to occipital dermoid cyst with dermal sinus: case report. Surg Neurol. 2002 Sep-Oct;58(3-4):266-70.

2. Ogle W. Cases illustrating the formation of morbid growths, deposits, tumors, cysts, etc, in connection with the brain and spinal cord and their membranes. Br For Med-Chir Rev. 1865; 36:200-58.

3. Layadi F, Louhab N, Lmejjati M, Aniba K, Ait Elqadi A, Ait Benali
S. Cerebellar dermoid cyst with occipital dermal sinus. Report of two pediatric cases. Pediatr Neurosurg. 2006;42(6):387-90.

4. Wright R. Congenital Dermal Sinuses. Prog Neurol Surg. 1971; 4:175-91.

5. Markus H, Kendall BE. MRI of a dermoid cyst containing hair. Neuroradiology. 1993;35(4):256-7.

6. Erdem G, Topcu M, Topaloglu H, Bertan V, Arikan U. Dermoid tumor with persistently low CSF glucose and unusual CT and MRI findings. Pediatr Neurol. 1994 Feb;10(1):75-7.

7. Soto-Ares G, Vinchon M, Delmaire C, Pruvo JP, Vallee L, Dhellemes P. Report of eight cases of occipital dermal sinus: an update, and MRI findings. Neuropediatrics. 2001 Jun;32(3): 153-8.

8. Groen RJ, van Ouwerkerk WJ. Cerebellar dermoid tumor and occipital meningocele in a monozygotic twin: clues to the embryogenesis of craniospinal dysraphism. Childs Nerv Syst. 1995 Jul;11(7):414-7.

9. Logue V, Till K. Posterior fossa dermoid cysts with special reference to intracranial infection. J Neurol Neurosurg Psychiatry. 1952 Feb;15(1):1-12.

10. Guidetti B, Gagliardi FM. Epidermoid and dermoid cysts. Clinical evaluation and late surgical results. J Neurosurg. 1977 Jul;47 (1):12-18.

11. Martinez-Lage JF, Ramos J, Puche A, Poza M. Extradural dermoid tumours of the posterior fossa. Arch Dis Child. 1997 Nov;77 (5):427-30.

12. Hashmi S, Jones RA. Delayed recurrence of cerebellar abscess 20 years after excision of dermoid cyst and sinus. Br J Neurosurg. 1998 Aug;12(4):358-60.

13. Schijman E, Monges J, Cragnaz R. Congenital dermal sinuses, dermoid and epidermoid cysts of the posterior fossa. Childs Nerv Syst. 1986;2(2):83-9.

14. Tanaka R, Miyasaka Y, Yada K, Kan S, Ichikawa F. Anomaly of venous system in congenital occipital dermal sinus. Acta Neurochir (Wien). 1994;128(1-4):174-9.

15. Yasargil MG, Abernathey CD, Sarioglu AC. Microneurosurgical treatment of intracranial dermoid and epidermoid tumors. Neurosurgery. 1989 Apr;24(4):561-7. 